\title{
A Commitment to Excellence
}

\author{
Wendy C. Budin, PhD, RN-BC, LCCE, FACCE, FAAN
}

\begin{abstract}
In this column, the editor of The Journal of Perinatal Education describes two international credentialing programs, the Magnet Recognition Program and the Baby-Friendly Hospital Initiative. Both require an ongoing commitment to excellence. The editor also describes the contents of this issue, which offer a broad range of resources, research, and inspiration for childbirth educators in their efforts to promote, support, and protect natural, safe, and healthy birth.
\end{abstract}

The Journal of Perinatal Education, 21(1), 3-5, http://dx.doi.org/10.1891/1058-1243.21.1.3

Keywords: normal birth, natural birth, safe birth, healthy birth, childbirth education, perinatal education, Wendy C. Budin Magnet recognition, Baby-Friendly

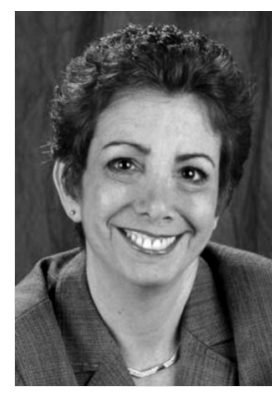

I recently returned from the 2011 American Nurses Credentialing Center's (ANCC's) National Magnet Conference where more than 7,000 nurses gathered to hear presentations and view posters celebrating excellence in nursing and health care. The ANCC's Magnet Recognition Program recognizes healthcare organizations for quality patient care, nursing excellence, and innovations in professional nursing practice. Consumers rely on Magnet designation as the ultimate credential for high quality nursing. Developed by the ANCC (2011), Magnet is the leading source of successful nursing practices and strategies worldwide. According to Craig Luzinski (2011), director of the Magnet Recognition Program, "It is not a prize or award-it is a performance-driven recognition credential that brings both external prestige and wide-ranging internal benefits" (p. 389). In addition to the benefits of Magnet status on outcomes such as quality improvement, safety, nurse engagement, satisfaction, and retention, the challenging process of pursuing and achieving Magnet recognition is noteworthy in that it requires a culture shift throughout the health-care organization demonstrating a commitment to excellence.

Similarly, when a hospital makes the commitment to implement the "Ten Steps to Successful Breastfeeding" required for achieving Baby-Friendly designation, as outlined by UNICEF/WHO (BabyFriendly USA, 2010a), a culture shift is required within the entire maternity department as well as support from the hospital administration. As stated by Baby-Friendly USA (2010b),

The Baby-Friendly Hospital Initiative (BFHI) is a global program sponsored by the World Health Organization (WHO) and the United Nations Children's Fund (UNICEF) to encourage and recognize

When a hospital makes the commitment to implement the "Ten Steps to Successful Breastfeeding" required for achieving Baby-Friendly designation, a culture shift is required within the entire maternity department. 
For more information about Baby-Friendly USA and implementing the UNICEF/ WHO Baby-Friendly Hospital Initiative, visit http://www. babyfriendlyusa.org/
The content of all JPE issues published since October 1998 is available on the journal's Web site (http:// www.ingentaconnect. com/content/springer/ jpe). Lamaze International members can access the site and download free copies of JPE articles by logging on at the "Members Only" link on the Lamaze Web site (www.lamaze.org). hospitals and birthing centers that offer an optimal level of care for infant feeding. The BFHI assists hospitals in giving mothers the information, confidence, and skills needed to successfully initiate and continue breastfeeding their babies or feeding formula safely, and gives special recognition to hospitals that have done so. (para. 4)

Recently, the hospital where I work (NYU Langone Medical Center, a Magnet hospital redesignated in 2009) became one of the 119 U.S. health-care facilities to earn the prestigious Baby-Friendly hospital designation. This lengthy journey requires the staff to thoroughly evaluate their current practices and adopt policies and procedures that lead to improved health outcomes for mothers and babies. To achieve the Baby-Friendly hospital designation, the healthcare facility must complete all of the requirements and ultimately demonstrate during an on-site assessment that the facility has correctly integrated all of the "Ten Steps To Successful Breastfeeding" into its practice for healthy newborns (Baby-Friendly USA, 2010c). I congratulate NYU Langone Medical Center on this outstanding achievement that demonstrates their ongoing commitment to excellence.

\section{IN THIS ISSUE}

In each issue of The Journal of Perinatal Education (JPE), we feature a special birth story to help advance the Lamaze International mission to promote, support, and protect natural, safe, and healthy birth. In this issue's "Celebrate Birth!" column, we share a story written by Jennifer DeVries, a second-time mother who relates her experience of birthing her son at home after her daughter was born via cesarean surgery. Support from the International Cesarean Awareness Network, as well as a home birth midwife specializing in vaginal birth after cesarean (VBAC), made her dream of a vaginal birth a reality. DeVries's story highlights the importance of having a supportive care provider and laboring in a safe and comfortable environment when pursuing a VBAC.

In a guest editorial, Dr. Raymond De Vries (uncle of Jennifer DeVries) shares an excerpt from an address he presented on the occasion of his inauguration as professor of Midwifery Science at University of Maastricht, The Netherlands, in May 2011. De Vries eloquently describes how, when done well, maternity care brings together four "voices": the voice of midwifery, which sees birth as a healthy physiological process; the voice of obstetrics, which can manage pathology; the voice of fear (or respect), which encourages vigilance; and the voice of trust, which allows a woman to have confidence in herself and her caregivers. $\mathrm{He}$ proposes that our goal is to keep these voices singing in harmony.

This issue's four featured articles cover a variety of topics of interest to perinatal educators and health-care providers. Swedish authors Ahldén, Ahlehagen, Dahlgren, and Josefsson present findings from their cross-sectional study of 1,117 pregnant women and 1,019 partners designed to assess parents' expectations about participating in antenatal parenthood education classes and to determine whether expectations were related to gender, age, and educational level. Participants believed that antenatal education classes would help them feel more secure as parents and be better oriented toward childbirth. Partners had more positive expectations about the childbirth than the mothers. The participants mostly wanted help in preparing for parenthood and in learning infant care skills, followed by help in preparing for childbirth. The participants' expectations were affected by gender, age, and educational level. Results indicated a need for improvement in antenatal classes to make these classes more attractive and to meet the requirements of today's expectant parents.

In their commentary article, Barnes, Roiko, Reed, Williams, and Willcocks discuss how assisted conception is becoming an increasingly more common treatment option for women and couples who experience fertility problems. The authors describe the links between assisted conception and a greater incidence of pregnancy or birth complications, low birth weight or premature babies, and babies with congenital abnormalities. In addition, the authors note that evidence suggests the experience of assisted conception may influence the development of early mothering relationships and impact parenting adjustment. Barnes et al's commentary article provides an overview of the health issues that women and families undergoing assisted conception have experienced or may experience. Through raising awareness and promoting discussion of these issues, the authors suggest that practitioners will be better equipped to provide informed education and support.

Hidaka and Callister present findings from their qualitative descriptive study that explored the birth experiences of women using epidural analgesia for pain management. Five themes emerged: (a) coping with pain, (b) finding epidural administration 
uneventful, (c) feeling relief having an epidural, (d) experiencing joy, and (e) having unsettled feelings of ambivalence. The authors suggest that although epidural analgesia was found to be effective for pain relief and may contribute to some women's satisfaction with the birth experience, it does not guarantee a quality birth experience. To support and promote childbearing women's decision making, Hidaka and Callister recommend improved education on the variety of available pain management options, including their risks and benefits. They also propose that fostering a sense of caring, connection, and control in women is a key factor to ensure positive birth experiences, regardless of pain management method.

In their article "Support Needs of Expectant Mothers and Fathers: A Qualitative Study," Swedish authors Widarsson, Kerstis, Sundquist, Engström, and Sarkadi describe the results of their qualitative study on expectant mothers' and fathers' perceived needs of support during pregnancy. Parents described not only a broad spectrum of social support needs, but also needs of psychological and physical support. Mothers' needs were predominately addressed in the health services, but fathers often felt "invisible." Widarsson et al. suggest that antenatal services may need to offer more customized individual support and emphasize peer support in groups.

In this issue's "Navigating the Maze" column, Judith Lothian explores current understandings of risk and safety in pregnancy and childbirth. Lothian suggests that an emphasis on risk management places the provider and hospital in control of women's decisions related to pregnancy and birth and may make pregnancy and birth less safe for mothers and babies. She proposes that by accepting that no life is risk free, women can let go of fear and make choices that take into account real, not imagined, or exaggerated risk and, in doing so, increase safety for themselves and their babies. With this perspective, the focus of maternity care becomes enhancing safety through evidence-based practice rather than managing risk.

In this issue's "Tools for Teaching” column, Barbara Hotelling presents information from three prominent Lamaze childbirth educators and from the literature to describe a variety of options that educators can share with expectant parents regarding the use of pain relief medications during labor and birth. For example, Ann Tumblin teaches about epidurals in a hospital class without losing sight of evidence-based practices that support normal birth. Jessica English focuses her classes on the natural processes of giving birth and spends only a little time presenting information about pain medications. Judith Lothian encourages educators to consider a new framework for Lamaze classes that involves letting go of the details and incorporating Lamaze's six Healthy Birth Practices and storytelling.

In this issue, JPE columnist Kimmelin Hull reprises a recent selection from the Lamaze International research blog, Science \& Sensibility. With the introduction of a new technology intended to acutely track fetal station and position during labor through birth, questions are being raised about the necessity - and possible danger - of this technology and its potential impact on normal birth.

In this issue's "Verse and Vision" column, entitled "Singing Along the Way to Becoming Baby-Friendly," two assistant nurse managers, Gladys Vallespir Ellett and Ieda delos Reyes, describe an innovative and fun strategy they developed and implemented at NYU Langone Medical Center in New York to assist the nursing staff in preparing for their successful auditors' visit on the road to become a Baby-Friendly hospital.

\section{REFERENCES}

American Nurses Credentialing Center. (2011). ANCC Magnet Recognition Program ${ }^{\circledR}$. Retrieved from http:// www.nursecredentialing.org/Magnet.aspx

Baby-Friendly USA. (2010a). BFHI USA-Implementing the UNICEF/WHO Baby Friendly Hospital Initiative in the U.S. About BFHI. The ten steps to successful breastfeeding. Retrieved from http://www.babyfriendlyusa. org/eng/10steps.html

Baby-Friendly USA. (2010b). BFHI USA-Implementing the UNICEF/WHO Baby Friendly Hospital Initiative in the U.S. About the BFHI. What is the Baby-Friendly Hospital Initiative and why do we need it? Retrieved from http://www.babyfriendlyusa.org/eng/01.html

Baby-Friendly USA. (2010c). BFHI USA-Implementing the UNICEF/WHO Baby Friendly Hospital Initiative in the U.S. Info for hospitals and birth centers. How do facilities go about achieving the Baby-Friendly designation? Retrieved from http://www.babyfriendlyusa.org/ eng/04.html

Luzinski, C. (2011). Introducing the official leadership journal of the Magnet Recognition Program ${ }^{\circledR}$. The Journal of Nursing Administration, 41(10), 389-390. http://dx.doi.org/10.1097/NNA.0b013e31822edb79

WENDY C. BUDIN is the editor-in-chief of The Journal of Perinatal Education. She is also the director of nursing research at NYU Langone Medical Center and a clinical professor at New York University, College of Nursing. She is a fellow in the American College of Childbirth Educators and is a member of the Lamaze International Certification Council. 